\title{
INDUSTRIAL DESIGN AND CLOUD-BASED 3D VISUALIZATION TOOL - AUTODESK FUSION 360
}

\author{
Rajan S. Pradhan \\ Department of Management Science \\ Dr. Babasaheb Ambedkar Marathwada Univeristy, Aurangabad
}

\begin{abstract}
The objective of this study is to provide review of industrial design and cloud-based 3D Visualization tool. This paper deals with introduction of industrial design. In order to exemplify and provide an overview of Autodesk Fusion 360 and its features useful in industrial design with models design by the author.
\end{abstract}

Keywords - Industrial Design, Visualization, 3D Modelling, cloud-based 3D modeling, Fusion 360, CAD, Rendering

\section{INTRODUCTION}

A design process is the series of activities by which the information about the designed object is changed from one information state to another. That is, a design process solves, or resolves, a design problem. Industrial design method typically features a two-graded structure. The first grade is the 'design process based on the designer's knowledge', and the second is the 'process based on the organizational knowledge.' Industrial design is a discipline that innovates the future. It is part of the industrial designer's job to identify trends, opportunities, social dynamics and technologies that no one else sees, and combine them in a new and useful product or service. And although stylish and expensive products for an exclusive public are part of industrial design, the field of study casts its net much, much wider [1].

Industrial Design links innovation, technology, research, business, and customers to provide new value and competitive advantage across economic, social, and environmental spheres Industrial design is concerned with the appearance and usefulness of manufactured goods. When creating products, industrial designers think about technical performance, environmental concerns, human comfort, and aesthetics [2]. Wikipedia defines Industrial Design as "the use of both applied art and applied science to improve the aesthetics, design, ergonomics, functionality, and/or usability of a product, and it may also be used to improve the product's marketability and even production. Industrial Design is a trans-disciplinary profession with strategic problem-solving processes that drive innovation, build business success, and cause a higher quality of life for the user through innovative products, systems, services, and experiences. As a result, the user should obtain a product cause a higher that has taken into account the user's needs in its everyday life.

Industrial design studies performs and form-and the association between product, user, and environment. Generally, industrial design professionals work in small scale design, instead of overall design of advanced systems such as buildings or ships. Industrial designers don't sometime design motors, electrical circuits, or gearing that make machines move, however they'll have an effect on technical aspects through usability design and form relationships. Usually, they work with other professionals such as engineers who design the mechanical aspects of the product assuring functionality and manufacturability, and with marketers to identify and fulfil client desires and expectations. "The work of a good designer with his product is not to force an ideal, but to generate an idea that was there waiting to come out. If he succeeds, the person will grow up to become his faithful user. If it were a meal, I would describe the taste, the color, the temperature, the ethnic origins, etc. However, he has not declared the ingredients or how they can arm themselves, that's the menu, his job is to find the menu that satisfies the hungry customer" [3]. The client starts with a set of requirements and conditions for the product and then asks the industrial designer to help consider about everything from how will customers impression when they look at the product to what can be added or taken away from the product to make it more user-friendly.

Industrial designers don't just think about the physical design of the product, they also need to understand the visual, safety, and convenience needs of the consumer as well as the technical requirements the manufacturer needs to build and market the product at scale, and they need to make sure that their design recommendations comply with all legal and regulatory requirements. The actual design process is different for different industrial designers but there is plenty of overlap. Almost all industrial designers understand the importance of doing research on the intended consumer, doing research on similar products already in the market, and prototyping or testing the product before distributing final references. Almost 


\section{International Journal of Engineering Applied Sciences and Technology, 2019 \\ Vol. 4, Issue 4, ISSN No. 2455-2143, Pages 36-40 \\ Published Online August 2019 in IJEAST (http://www.ijeast.com)}

all industrial designers will also sketch or model their designs and that is where different designers use different processes. Some designers prefer traditional pen and paper sketches on loose-leaf paper, but with the advancement of technology, more and more designers are beginning to utilize things like 3D modeling software, computer-aided design tools, and CAD programs.

The objective of data visualization is to support humans in understanding and acting upon data values, or analytics result from data values. Basically the process creates ideas in the mind using images, we believe there are three main roles for visualization:

Communication: presenting, describing and explaining ideas, examples of which might be education and training.

Confirmation: testing hypothesis, monitoring information streams, examples of which might be scientific results and internet of things streaming data.

\section{A. Cloud based 3D visualization}

$3 \mathrm{D}$ visualization is the process of creating graphics and rendering designs using 3D software. Many industries benefit from $3 \mathrm{D}$ visualization ranging from architecture, film, and games, to engineering and manufacturing. [4] A number of products and visions are created in $3 \mathrm{D}$ visualization prior to execution, saving both time and costs for producers, manufacturers and customers.

3D CAD modelling tools especially have been beneficial to increasing the speed with which product engineers can generate models for tooling, prototyping, patenting, marketing, and manufacturing. 3D CAD modelling software tools, and Industrial designer chose 3D CAD modelling software tools that have the most useful features and best suited for visualization and quick ideation and rendering, while others are useful for freeform surface modelling. Now a days available 3D CAD Modelling Tools are Tinkercad, Solidworks, AutoCAD, FreeCA like D, TurboCAD 2016 Deluxe, Creo Parametric 3D , Modeling Software, IronCAD, Moment of Inspiration, Shapr3D, Fusion 360, BricsCAD, OpenSCAD , VariCAD, SolveSpace, Blender, BRL-CAD, Mesh mixer, DesignSpark Mechanical, Inventor, Rhino, Patchwork 3D, Onshape for Professional Design Teams, KeyCreator Direct CAD, Solid Edge ST9, SketchUp, AutoCAD Mechanical, SpaceClaim,DesignCAD 3D Max, CATIA 3DEXPERIENCE, 3DCrafter, Creo Elements/Direct, MeshLab, CMS IntelliCAD, ZW3D, SolidFace Collaborative Cloud 3D CAD Software, KOMPAS-3D, NX for Product Design, AC3D, K3D, Geomagic Design, ZBrush 4R7, Vectorworks Fundamentals, 3DS Max, Modo, Autodesk Alias, OpenJSCAD, ImplicitCAD, Antimony, Strata Design 3D and Morphi etc for Ideation, Rendering, Prototyping and More for Industrial Engineers.

\section{B. Fusion 360}

A cloud-powered 3D CAD software tool for product engineers, Fusion 360 is a 3D CAD, CAM, and CAE tool in an association of its own. Fusion 360 connects the entire product development process in a single cloud-based platform that works on Macs and PCs. Fusion 360 is a fully featured parametric cadet program that has nearly all of the features one would find in costly professional CAD software. Fusion 360 makes a complete set of tools available to the user. Aside from the standard modeling tools, Fusion 360 also includes environments for creating surfaces and sheet metal parts. When it comes time for testing, there's a simulation environment with features such as static stress and thermal analysis. The manufacturing side of things is rounded out by a robust set of aids for computer aided manufacturing (CAM). Fusion 360, a product introduced by Autodesk and integrating industrial design, structural design, mechanical simulation, and CAM, turns out a design platform supporting collaboration and sharing. Both cross-platform and via the cloud.

Fusion 360 is, at its core, Autodesk's cloud-enabled mechanical 3D-modeling tool. [5] And it's a fantastic one at that, offering much more than the common capabilities of traditional 3D CAD (cloud and otherwise) - capabilities such as free-form modeling and sculpting with T-Splines, mesh modeling, powerful direct modeling and parametric modeling, and an API to enable third-party extensibility. A suite of tools for rendering, creating animations, renderings and drawings rounds out the toolbox, ensuring that nearly every aspect of design is covered.

\section{MODELING}

The existence of CAD based design systems are saviours in many sections of the market. In such systems, it is possible to see the finished product before it is produced, and therefore, it have increasingly been preferred, by most of the design teams and manufacturers. 3D design software helps designer in having a much better idea about the performance of the device he designed. It not only reduces time but also helps the designer to see the end product in a reliable way. Appearance models do not function but are intended to show what a product will look like when it is produced, where, the functional models may not look like the end product, but they are operational. These are usually used to work out the mechanical or electronic systems.

Nowadays, a designer can wear many hats. Depending on where you work, you might not only be making 3D models to prototype ideas, but also making $3 \mathrm{D}$ models to send to manufacturing companies to produce molds for production. Having a good understanding of CAD will not only help you test your ideas but also take them all the way to the final goal: real-world production. [6] 


\section{International Journal of Engineering Applied Sciences and Technology, 2019 \\ Vol. 4, Issue 4, ISSN No. 2455-2143, Pages 36-40 \\ Published Online August 2019 in IJEAST (http://www.ijeast.com)}

A salient feature of the direct modeling in Fusion360 is the visibility of outcomes. The direct modeling technology enables users to Performa follow-up modeling up on models both featured and non-featured (such as the nonparametric models acquired by other CAD systems). In the whole process, any modification or addition makes no difference to the establishment of models. [7]

The future of CAD certainly means a combination of the accuracy and control of design with the speed and flexibility of precise modelling. The result is that designers spend less time planning a model's construction, they are more focused on waiting for the changes in design, and they spend less time in remodeling the design for future uses [8]

We can categorize today's 3D modeling software into two broad types: parametric modelling and mesh modeling. Although the two types often overlap, people generally use parametric modeling software for engineering purposes and mesh modeling software for artistic purposes. [9]

Parametric modeling in fusion 360: The feature-based parametric modeling technique enables the designer to incorporate the original design intent into the construction of the model. The word parametric means the geometric definitions of the design, such as dimensions, can be varied at any time in the design process. Parametric modeling is accomplished by identifying and creating the key features of the design with the aid of computer software. [10] The design variables, described in the sketches as parametric relations, can then be used to quickly modify/update the design. [11]

Autodesk Fusion 360 provides many powerful modeling and design-tools, and there are many different approaches to accomplishing modeling tasks.

Parametric modelling is suitable for the modelling of parts with functional geometries (mechanical parts, such as a gear, for example). Models are then further refined by parameters which can be easily modified.

Direct modelling offers more flexibility in terms of creativity and aesthetics. In fact, by manipulating curves and surfaces one can obtain very beautiful pieces with complex and organic shapes.

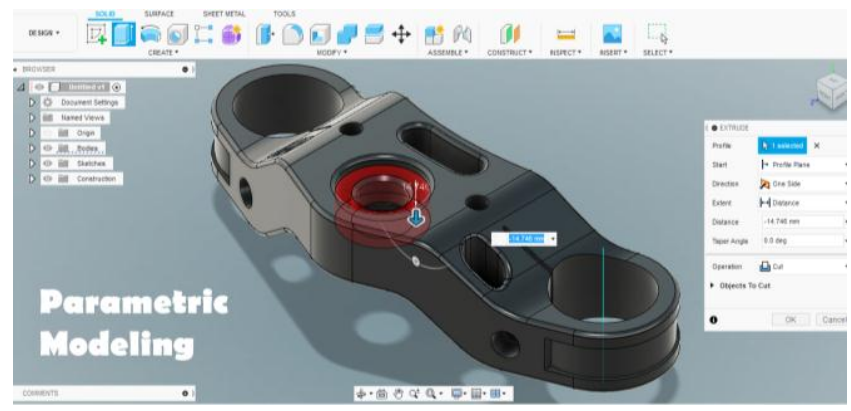

Fig. 1 Parametric Modelling
Fusion 360 operates on a spline system (curves passing through fixed points), and more precisely T-spline. The fundamental difference between T-splines and NURBS surfaces is the presence of star points. The star points allow to create non-rectangular polygons and thus very complex curved surfaces by limiting the quantity of polygons on the mesh.

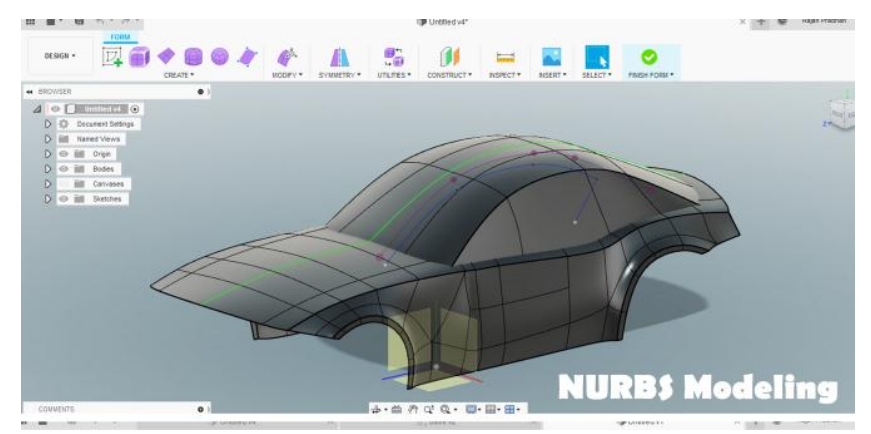

Fig. 2 NURBS Modelling

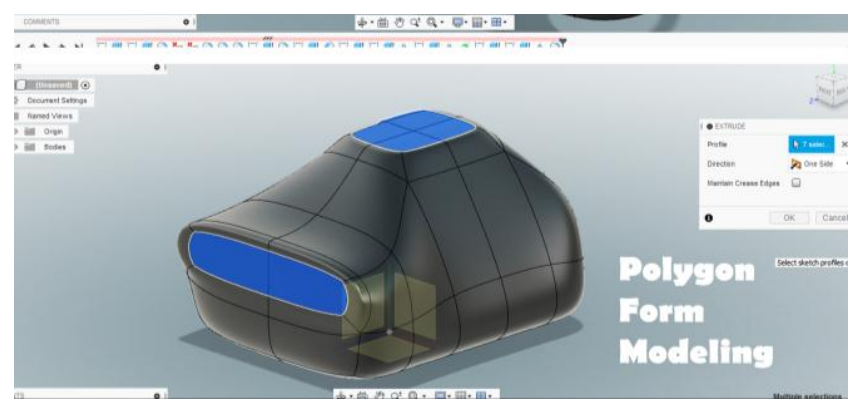

Fig. 3 Polygon Form Modelling

Fusion 360 supports several ways of designing assemblies. In the process of assembly design, a project manager, once being imported necessary information in, can automatically generate the list of assembly parts, and realize the driven assembly of mechanical components under constraints. When assembling parts in the same design environment, we can assemble parts based on the same coordinate point, and set various types of joints, such as swivel, sliders, cylinders, pin troughs, and spheres. The user can also set the movement limit of the joint and make precise configuration of the numerical settings for moving, so as to realize a real-time preview of the movement. Fusion 360 assembly commands render a very clear picture of the connection between parts, and help produce assembly animation when referring the animation modules. 


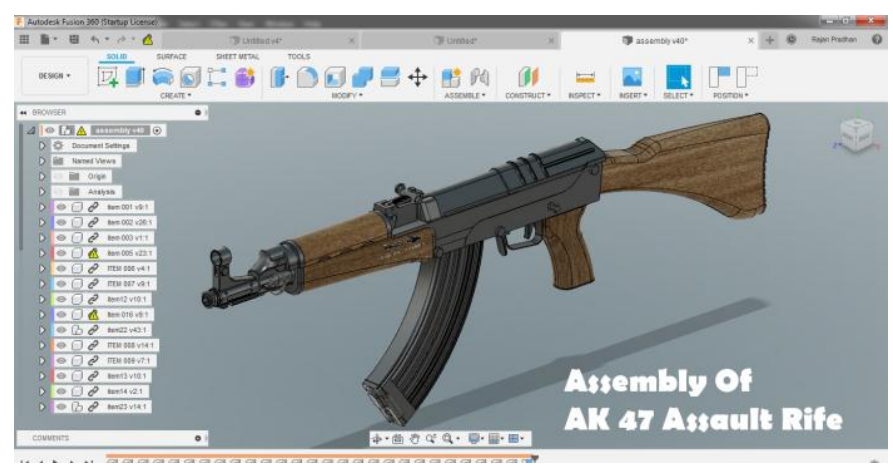

Fig. 4 Assembly Design

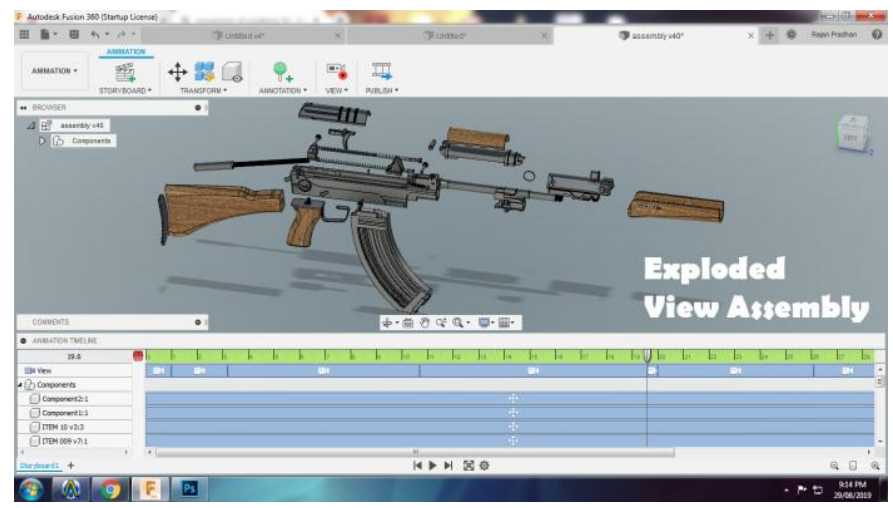

Fig. 5 Exploded view Assembly

\section{RENDERING}

Rendering is that the method of processing an image by combining geometry, camera, texture, lighting and shading (also referred to as materials) information using a computer program. Before an image can be rendered Appearance Materials are applied to the various parts of your design to visualize how your design would look in the real word. Materials contain the visual properties of plastic, glass, metal, paint and wood (and pretty much anything else you can think of) to create photorealistic images.

Fusion 360 is no exception. In addition to a huge rendering material database, the current version has two rendering methods, local Ray Tracing rendering, and cloud Showcase 360 rendering. Fusion360 also supports the inner rendering of canvas, a process stating to the real-time rendering of scenes. Fusion360 would perform continuous rendering and iteration, which progressively renders the rough scene delicate.

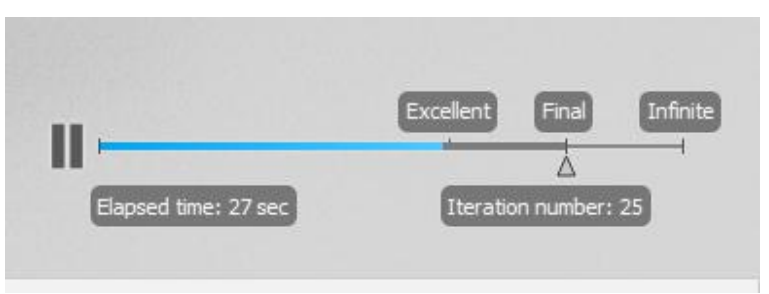

Fig. 6 Rendering progess (veiw of action bar for rendering time, progress, iteration, and quality.)

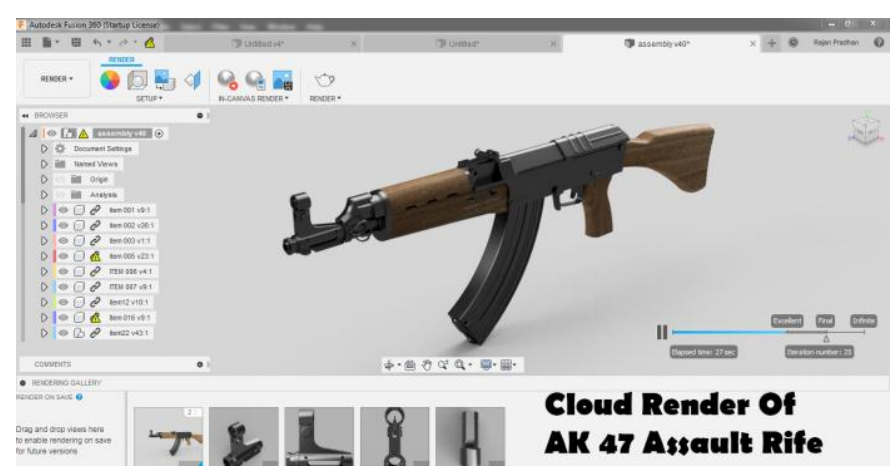

Fig. 7 Cloud Rendering

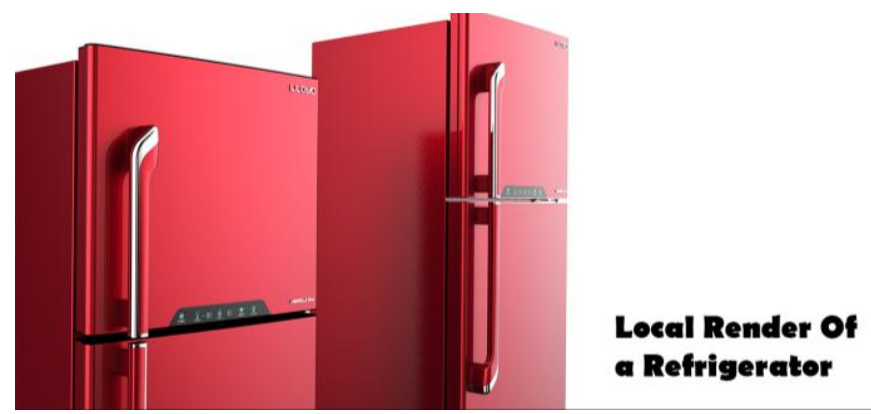

Fig. 8 Local Rendering

Fusion 360 assembly commands render a very clear picture of the relationship between parts, and help produce assembly animation when consulting the animation modules.[12] The assembly system in Fusion360 provides seven kinds of contact movement: rigid, rotating, sliding, cylinder, pin slot, plane, and ball.

\section{CONCLUSION}

In summary, Fusion 360 is ideal to work with industrial design all along the innovation, design and development in order to reach a final product that is globally competitive, technologically innovative and, most of all, formed around the user's capabilities and goals.

\section{ACKNOWLOGEMENT}

The author would like to acknowledge the support of the Arrow Design Studio, Aurangabad (MH) India.

\section{REFERENCE}

[1] Baelus Chris (2015) Research In Flanders- Thematic papers- Industrial Design (Pg. 3-7)

[2] http://www.idsa.org/news/dblog/what-id

[3] Ogrodnik P. (2015) Class 1 Devices Case Studies in Medical Devices Design. ISBN: 978-0-12-800796-9

[4] http://thewaytodream.com/design-3d-modelingvisualization/

[5] Tamburini Diego (2015). Delivering the future of making things with fusion 360. Autodesk-2015

[6] http://jordanpelovitz.com/modeling-for-industrial-design/ 
[7] Song P P. (2018). Research and Application of Autodesk Fusion360 in Industrial Design, IOP Publishing. DOI:10.1088/1757-899X/359/1/012037

[8] Akca Enes (2017) Development of Computer-Aided Industrial Design Technology. Periodicals of Engineering and Natural Sciences Vol.5, No.2 (pp. 124-127) ISSN 2303-4521 DOI: 10.21533/pen.v5i2.86

[9] Coward Cameron (2019) A Beginner's Guide to 3D Modeling: A Guide to Autodesk Fusion 360 ISBN : 978-1-59327-927-4

[10] Shih Randy H. Parametric Modeling: Pro/Engineer Wildfire 2.0, ISBN: 1585031887, 9781585031887

[11] Shih Randy H.(2017) Learning Autodesk Inventor 2018 Modeling, Assembly and Analysis. ISBN10: 1630571318, ISBN-13: 978-1630571313

[12] https://www.lynda.com/Fusion-360-tutorials/Fusion360-Animating-Assemblies/740362-2.html 